\title{
Antimycin A sensitizes cells to TRAIL-induced apoptosis through upregulation of DR5 and downregulation of c-FLIP and Bcl-2
}

\author{
SUNG-JUN LEE ${ }^{1}$, EUN-AE KIM ${ }^{1}$, KYOUNG SEOB SONG ${ }^{3}$, MIN-JAE KIM ${ }^{1}$, \\ DAE HYUNG LEE ${ }^{2}$, TAEG KYU KWON ${ }^{4}$ and TAE-JIN LEE ${ }^{1}$
}

\author{
Departments of ${ }^{1}$ Anatomy, College of Medicine and ${ }^{2}$ Obstetrics and Gynecology, Yeungnam University, Daegu; \\ ${ }^{3}$ Department of Physiology, College of Medicine, Kosin University, Busan; ${ }^{4}$ Department of Immunology, \\ School of Medicine, Keimyung University, Daegu, Republic of Korea
}

Received May 4, 2012; Accepted July 2, 2012

DOI: 10.3892/ijo.2012.1575

\begin{abstract}
Tumor necrosis factor-related apoptosis-inducing ligand (TRAIL) has been the focus as a potential anticancer drug, because it induces apoptosis in a wide variety of cancer cells but not in most normal human cell types. In this study, we showed that combination treatment with sub-toxic doses of antimycin A (AMA), an inhibitor of electron transport, plus TRAIL induced apoptosis in human renal cancer cells, but not in normal tubular kidney cells. Treatment of Caki cells with AMA upregulated the death receptor 5 (DR5) protein and downregulated c-FLIP and $\mathrm{Bcl}-2$ proteins in a dose-dependent manner. AMA-induced decrease of c-FLIP $\mathrm{L}_{\mathrm{L}}$ and c-FLIPs protein levels which were caused by increased protein instability, which was confirmed by the result showing that treatment with a protein biosynthesis inhibitor, $\mathrm{CHX}$, accelerated degradation of c-FLIP $\mathrm{L}_{\mathrm{L}}$ and c-FLIPs proteins caused by AMA treatment. We also found that AMA induced upregulation of DR5 and downregulation of Bcl-2 at the transcriptional level. Pretreatment with N-acetyl-1-cysteine (NAC) partly recovered the expression levels of c-FLIP $\mathrm{L}_{\mathrm{L}}$ and c-FLIPs proteins were downregulated by the AMA treatment, suggesting that AMA appears to be partially dependent on the generation of ROS for downregulation of c-FLIP ${ }_{\mathrm{L}}$ and c-FLIPs. Collectively, this study demonstrates that AMA enhances TRAIL-induced apoptosis in human renal cancer cells by upregulation of DR5 as well as downregulation of c-FLIP and Bcl-2. Furthermore, this study shows that AMA markedly increases sensitivity to cisplatin in Caki human renal cancer cells.
\end{abstract}

\section{Introduction}

Tumor necrosis factor (TNF)-related apoptosis-inducing ligand (TRAIL) is considered as a new potential anticancer drug.

Correspondence to: Dr Tae-Jin Lee, Department of Anatomy, College of Medicine, Yeungnam University, 317-1, Daemyung-Dong Nam-Gu, Daegu 705-717, Republic of Korea

E-mail: tjlee@med.yu.ac.kr

Key words: antimycin A, renal cancer, tumor necrosis factorrelated apoptosis-inducing ligand, death receptor 5, Bcl-2, c-FLIP
TRAIL induces apoptosis in various types of cancer cells in vitro and in vivo, but has little or no toxicity against normal cells, which is supported by the presence of large numbers of decoy receptors on normal cells $(1,2)$. Since TRAIL is a tumorselective and apoptosis-inducing cytokine, it is considered to be a promising new candidate for cancer prevention and treatment. However, recent studies have shown that some cancer cells are resistant to the apoptotic effects of TRAIL (3-5). TRAIL-resistant cancer cells can be sensitized by chemotherapeutic drugs and biochemical inhibitors in vitro, indicating a possibility of combination therapy. Therefore, understanding the molecular mechanisms of TRAIL resistance and ways to sensitize these cells for apoptosis by TRAIL are important issues for effective cancer therapy.

Antimycin A (AMA) inhibits succinate oxidase and nicotinamide adenine dinucleotide (NADH) oxidase and also blocks mitochondrial electron transport by binding to complex III (6). Inhibition of electron transport causes the collapse of the mitochondrial proton gradient across the mitochondrial inner membrane and the mitochondria membrane potential (MMP), thus allowing production of reactive oxygen species (ROS) as well as the release of proapoptotic molecules such as cytochrome $c$ (7-11). Although a large number of studies have shown antitumor activity of AMA, anticancer effects of the combination of AMA and TRAIL are not clear. This study was designed to evaluate the combination treatment with AMA and TRAIL and to delineate the underlying mechanism associated with observations in vitro demonstrated by synergistic effects between AMA and TRAIL in renal cancer cells.

\section{Materials and methods}

Cells and materials. Caki cells were obtained from the American Type Culture Collection (ATCC, Rockville, MD, USA). Dulbecco's modified Eagle's medium (DMEM), containing 10\% fetal bovine serum (FBS), 20 mM HEPES buffer and $100 \mu \mathrm{g} / \mathrm{ml}$ gentamicin was the culture medium used throughout the experiments. AMA and TRAIL was directly added to cell cultures at the indicated concentrations. Anti-Bcl-2, anti-procaspase-3, anti-PARP and anti-actin antibodies were purchased from Santa Cruz Biotechnology Inc. (Santa Cruz, CA, USA). Anti-c-FLIP antibody was purchased from Alexis 
Corporation (San Diego, CA, USA). Soluble recombinant TRAIL was purchased from R\&D Systems (Minneapolis, MN, USA). AMA was obtained from Sigma Chemical Co. Anti-DR5 was purchased from Koma Biotech Inc. (Seoul, Korea).

Western blot analysis. Cellular lysates were prepared by suspending $1 \times 10^{6}$ cells in $100 \mu \mathrm{l}$ of lysis buffer $(137 \mathrm{mM} \mathrm{NaCl}$, $15 \mathrm{mM}$ EGTA, $0.1 \mathrm{mM}$ sodium orthovanadate, $15 \mathrm{mM} \mathrm{MgCl}_{2}$, $0.1 \%$ Triton X-100, $25 \mathrm{mM}$ MOPS, $100 \mu \mathrm{M}$ phenylmethylsulfonyl fluoride, and $20 \mu \mathrm{M}$ leupeptin, adjusted to $\mathrm{pH}$ 7.2). The cells were disrupted by sonication and extracted at $4^{\circ} \mathrm{C}$ for $30 \mathrm{~min}$. Then, the proteins were electrotransferred to Immobilon-P membranes (Millipore Corporation, Bedford, MA, USA). Detection of specific proteins was carried out with an ECL western blotting kit according to the manufacturer's instructions.

Flow cytometry analysis. Approximately $1 \times 10^{6}$ Caki cells were suspended in $100 \mu \mathrm{l}$ of PBS, and $200 \mu \mathrm{l}$ of $95 \%$ ethanol was added while vortexing. The cells were incubated at $4^{\circ} \mathrm{C}$ for $1 \mathrm{~h}$, washed with PBS, and resuspended in $250 \mu \mathrm{l}$ of $1.12 \%$ sodium citrate buffer ( $\mathrm{pH} 8.4$ ) together with $12.5 \mu \mathrm{g}$ of RNase. Incubation was continued at $37^{\circ} \mathrm{C}$ for $30 \mathrm{~min}$. The cellular DNA was then stained by applying $250 \mu \mathrm{l}$ of propidium iodide $(50 \mu \mathrm{g} / \mathrm{ml})$ for $30 \mathrm{~min}$ at room temperature. The stained cells were analyzed by fluorescent activated cell sorting (FACS) on a FACScan flow cytometer for relative DNA content based on red fluorescence.

RNA isolation and reverse transcriptase-polymerase chain reaction ( $R T-P C R)$. RT-PCR analysis was done as previously reported (12). The cDNA for DR5 and actin were amplified by PCR with specific primers. The sequences of the sense and antisense primers for DR5 were 5'-AAGACCCTTGTGCTCG TTGT-3' and 5'-GACACATTCGATGTCACTCCA-3', respectively. PCR products were analyzed by agarose gel electrophoresis and visualized by ethidium bromide. For CHOP, the sense primer 5'-CAACTGCAGAGATGGCAGCTGA-3' and the antisense primer 5'-CTGATGCTCCCAATTGTTCAT-3' (corresponding to a 536-bp region of $\mathrm{CHOP}$ ) were used. The sequences of the sense for c-FLIP ${ }_{\mathrm{L}}$ and c-FLIPs were 5'-CGGACTATAGAGTGCTG ATGG-3' and the antisense primers were 5'-GATTATCAGGCA GATTCCTAG-3' (c-FLIP ${ }_{\mathrm{L}}$ ) and 5'-AGATCAGGACAATGGG CATAG-3' (c-FLIPs), respectively.

Statistical analysis. Three or more separate experiments were performed. Statistical analysis was done by paired Student's t-test or ANOVA. A p $<0.05$ was considered to have pronounced difference between experimental and control groups.

\section{Results}

AMA sensitizes renal cancer cells for TRAIL-mediated apoptosis. In an attempt to search for novel strategies to overcome TRAIL resistance in cancer cells, we investigated the effect of combination treatment of AMA and TRAIL in human renal cancer cell line, Caki cells. Co-treatment of Caki cells with AMA and TRAIL resulted in a markedly increased in accumulation of sub-G1 phase cells, compared with Caki cells treated with AMA or TRAIL alone (Fig. 1A). In addition, combination treatment of Caki cells with AMA and TRAIL led to the reduction of the protein levels of procaspase 3. The protein levels of c-FLIP ${ }_{L}$,
c-FLIPs, Mcl-1 and Bcl-2 were remarkably reduced in response to combination of AMA plus TRAIL (Fig. 1B). Next, we analyzed nuclear condensation, which is another hallmark of apoptosis. Combination treatment with AMA plus TRAIL induced the nuclear condensation in Caki cells. However, we failed to detect nuclear condensation in Caki cells treated with TRAIL or AMA alone (Fig. 1C). Next, we investigated whether co-treatment with AMA and TRAIL affected TCMK-1 normal renal tubular epithelial cells. Interestingly, TCMK-1 cells were resistant to AMA or TRAIL alone, and cell morphology was not significantly affected by combination treatment with AMA and TRAIL (Fig. 1D).

Combination treatment with AMA plus TRAIL induced apoptosis is mediated via caspase-dependent pathway. We next examined whether activation of caspase pathway plays a critical role in AMA plus TRAIL-induced apoptosis. As shown in Fig. 2A, AMA plus TRAIL-induced apoptosis was completely prevented by pretreatment with the general and potent inhibitor of caspases, z-VAD-fmk, as determined by FACS analysis. We also found that $\mathrm{z}$-VAD-fmk prevented all these caspase-related events including cleavage of procaspase-3 and PARP (Fig. 2B). These results suggest that combined treatment of AMA and TRAIL-induced apoptosis was mediated by caspase-dependent apoptosis pathways.

AMA downregulates $c$-FLIP and Bcl-2 protein expressions but upregulates DR5 and CHOP proteins expression. To investigate the underlying mechanisms involved in combination treatment of AMA and TRAIL-induced apoptosis, we analyzed the expression levels of various apoptosis regulating proteins using western blot assay. While protein levels of c-FLIP ${ }_{\mathrm{L}}$, c-FLIPs, and Bcl-2 were noticeably reduced in response to AMA treatment, the levels of death receptor 5 (DR5) and CHOP proteins were increased by AMA treatment (Fig. 3A). Next, we examined whether AMA-mediation of c-FLIP $\mathrm{L}_{\mathrm{L}}, \mathrm{c}$-FLIPs and Bcl-2 downregulation were controlled at the transcriptional level. RT-PCR analysis demonstrated that AMA decreased Bcl-2 mRNA levels in a dosedependent manner. In addition, we found that DR5 and CHOP mRNA levels were increased by AMA treatment (Fig. 3B) in a dose dependent manner. However, c-FLIP ${ }_{\mathrm{L}}$ and c-FLIPs mRNA levels remained constant throughout the AMA treatment at different doses in Caki cells (Fig. 3B). These results suggest the possibility that AMA-mediated degradation of $\mathrm{c}-\mathrm{FLIP}_{\mathrm{L}}$ and c-FLIPs proteins might be regulated by post-transcriptional levels. To further clarify the underlying mechanisms of the decreased c-FLIP and c-FLIPs protein levels in AMA-treated cells, we analyzed the protein stability test of $\mathrm{c}-\mathrm{FLIP}_{\mathrm{L}}$ and c-FLIPs. Caki cells were treated with cycloheximide (CHX) and AMA at different doses. We found that the degradation of c-FLIP $_{\mathrm{L}}$ and c-FLIPs protein were facilitated by AMA treatment (Fig. 3C), which implies that AMA treatment caused reduction of c-FLIP protein stability. We also found that AMA-induced downregulations of $c-F_{L} P_{L}$ and $\mathrm{c}-\mathrm{FLIP}_{\mathrm{S}}$ proteins were partly blocked by pretreatment of anti-oxidant N-acetyl-L-cysteine (NAC) (Fig. 3D). These results suggest the possibility that AMA-stimulated downregulation of c-FLIP protein appears to be partly dependent on the generation of reactive oxygen species (ROS).

Downregulation of $c-F L I P_{L}$ and $c$-FLIPs also contributes to AMA-stimulated TRAIL-induced apoptosis. We exam- 

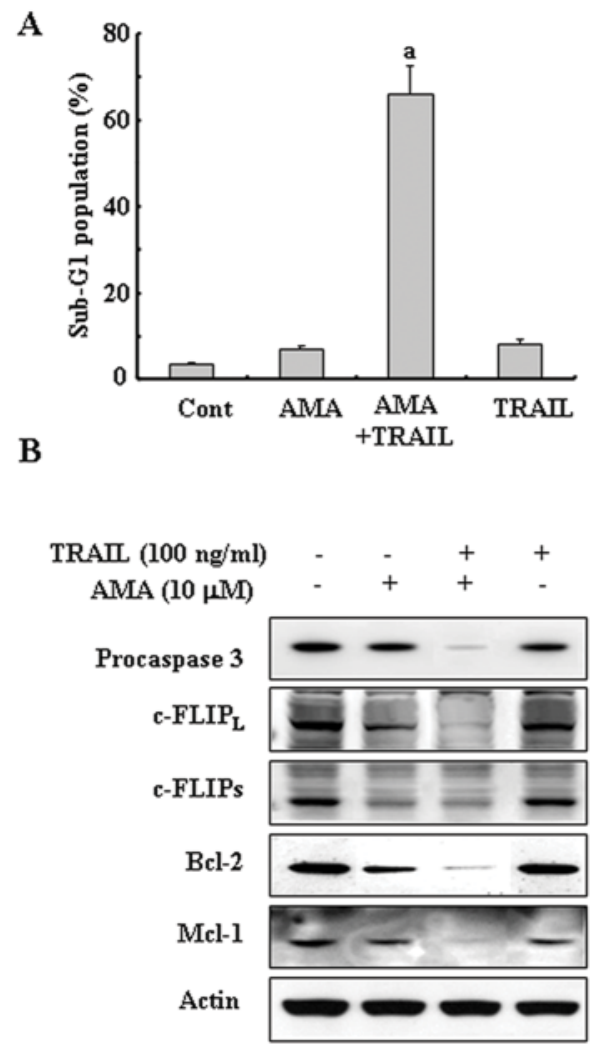
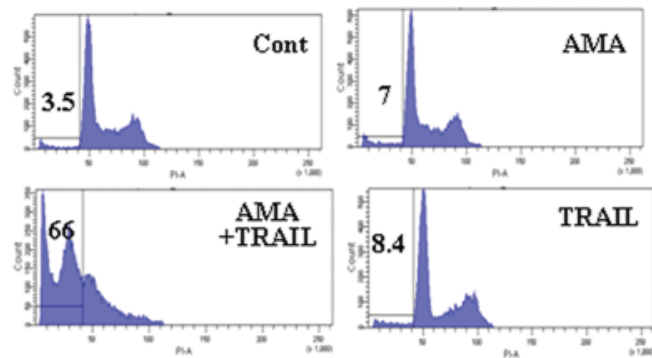

C
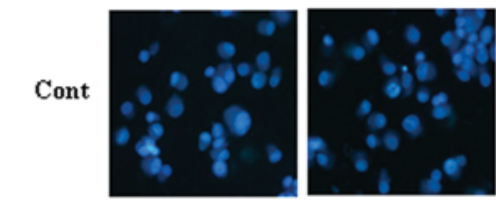

AMA
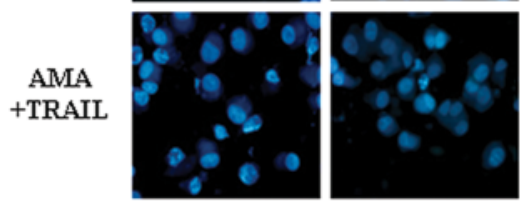

TRAII
D

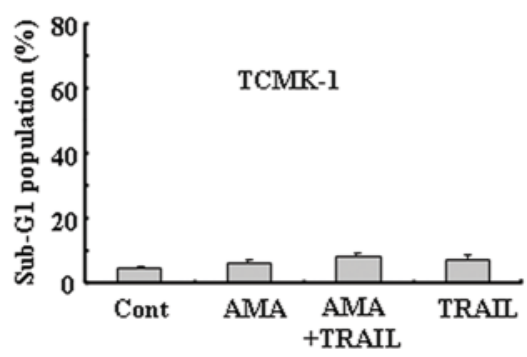

Figure 1. AMA sensitizes renal cancer cells to TRAIL-mediated apoptosis. (A) Caki cells were treated for $12 \mathrm{~h}$ with TRAIL (100 ng/ml) in either the absence or the presence of AMA $(10 \mu \mathrm{M})$. After $12 \mathrm{~h}$ of treatment, apoptosis was analyzed as a sub-G1 fraction by FACS. ${ }^{\mathrm{a}} \mathrm{p}<0.05$ for AMA+TRAIL-treated cells versus control, AMA- or TRAIL-treated cells by ANOVA. The FACS data are shown in the right panel. (B) Cells were treated with the indicated concentrations of AMA and TRAIL. Equal amounts of cell lysates $(40 \mu \mathrm{g})$ were subjected to electrophoresis and analyzed by western blotting for PARP, procaspase 3 , c-FLIP ${ }_{\mathrm{L}}$, c-FLIPs, Bcl-2 and actin for normalization. (C) After treatment with AMA plus and TRAIL for $12 \mathrm{~h}$, cells were harvested and washed with PBS and exposed to $300 \mathrm{nM}$ 4',6-diamidino-2-phenylindole (DAPI) at room temperature in the dark for $15 \mathrm{~min}$. Samples were observed under a fluorescence microscope. The healthy cell had an oval-shaped cell body, and its chromatin stained dimly and occupied the majority of the cell body. The chromatin of an apoptotic cell was condensed, intensely stained, or shifted to the periphery of the cell body. The morphology of cells were determined by interference light microscopy (magnification, x200). (D) Mouse tubular normal TCMK-1 cells were treated for $12 \mathrm{~h}$ with TRAIL $(100 \mathrm{ng} / \mathrm{ml})$ in either the absence or the presence of AMA $(10 \mu \mathrm{M})$. After $12 \mathrm{~h}$ of treatment, apoptosis was analyzed as a sub-G1 fraction by FACS.

ined whether downregulations of $\mathrm{c}-\mathrm{FLIP}_{\mathrm{L}}$ and c-FLIPs by AMA is critical in stimulating TRAIL-induced apoptosis. Overexpression of c-FLIP ${ }_{L}$ and c-FLIP ${ }_{S}$ in Caki cells significantly attenuated AMA-facilitated TRAIL-induced apoptosis, whereas combination treatment with AMA plus TRAIL induced significant apoptosis in Caki/vector cells (Fig. 4A). Cleavage of PARP and procaspase 3 induced by combination treatment were also markedly inhibited by the overexpression of c-FLIP and c-FLIP ${ }_{S}$ (Fig. 4B). These results suggest that c-FLIP ${ }_{L}$ and $\mathrm{c}^{-F L I P} \mathrm{~S}_{\mathrm{S}}$ downregulations also contribute to AMA-facilitated TRAIL-induced apoptosis.

AMA sensitizes renal cancer cells to cisplatin-mediated apoptosis. We also investigated whether the sensitivity against chemotherapeutic agent, cisplatin, increases in Caki cells. As shown in Fig. 5A, pretreatment with AMA augmented the sensitivity to cisplatin. In addition, combination treatment of Caki cells with AMA and cisplatin led to a reduction of the protein level of procaspase 3 together with a concomitant cleavage of PARP, a substrate protein of caspases (Fig. 5B).

\section{Discussion}

In this study, we demonstrated for the first time that combination treatment with AMA plus TRAIL on renal cancer cells synergistically induced apoptosis. AMA-induced upregulation of DR5 or AMA-mediated downregulation of Bcl-2 is controlled at the transcriptional level in a dose-dependent manner. In contrast, AMA-induced downregulation of $\mathrm{c}-\mathrm{FLIP}_{\mathrm{L}}$ and c-FLIPs is caused by facilitating degradation of c-FLIP protein. In addition, we also found that production of ROS by AMA treatment seemed to partially take part in $\mathrm{c}-\mathrm{FLIP}_{\mathrm{L}}$ and c-FLIPs downregulations.

Induction of DR5 expression is an important underlying mechanism for sensitization of TRAIL-mediated apoptosis (13-15). In this study, we detected the induction of CHOP mRNA and DR5 mRNA expressions in AMA-treated Caki cells, indicating the possible involvement of DR5 modulation in AMA-induced sensitization of TRAIL-prompted apoptosis in these cells. However, several reports have shown that CHOP upregulates DR5 expression at the transcriptional 


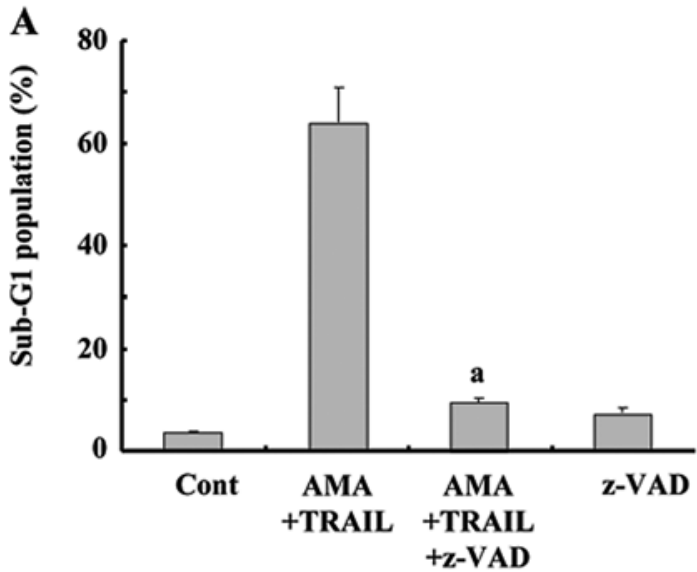

\section{B}

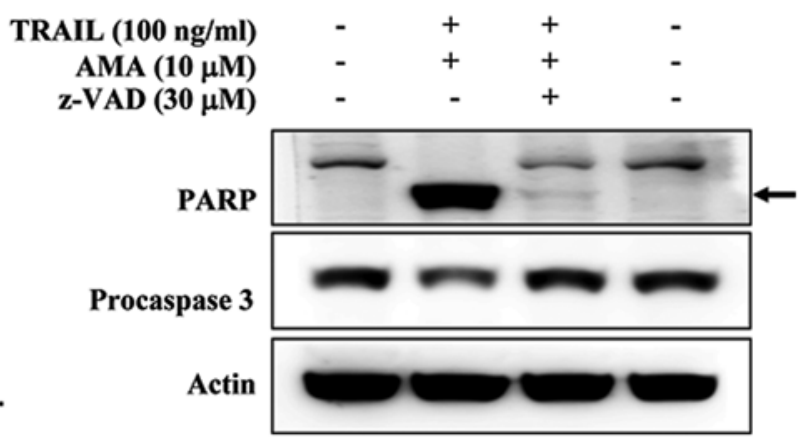

Figure 2. Combined treatment with AMA plus TRAIL induced apoptosis is mediated by caspase-dependent pathway. (A) Effect of z-VAD-fmk on apoptosis induced by AMA plus TRAIL. Caki cells were incubated with $50 \mu \mathrm{M}$ z-VAD-fmk or solvent for $1 \mathrm{~h}$ before treatment with AMA (10 $\mu \mathrm{M})$ and/or TRAIL (100 ng/ml) for $12 \mathrm{~h}$. DNA contents of treated cells were evaluated after propidium iodide staining and apoptosis was measured as a sub-G1 fraction by FACS. Data are mean values obtained from three independent experiments and bars represent standard deviation. ${ }^{a} \mathrm{p}<0.05$ compared to AMA plus TRAIL-treated cells. (B) Effect of $\mathrm{z}$-VAD-fmk on caspase activation in AMA plus TRAIL treated cells. Cells were treated with the indicated concentrations of AMA and TRAIL. Equal amounts of cell lysates $(40 \mu \mathrm{g})$ were subjected to electrophoresis and analyzed by western blotting for procaspase 3 and PARP. The proteolytic cleavage of PARP is indicated by an arrow. A representative study is shown; two additional experiments yielded similar results.

A

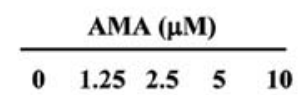

DR5

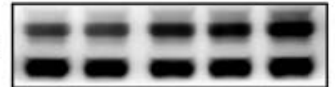

CHOP

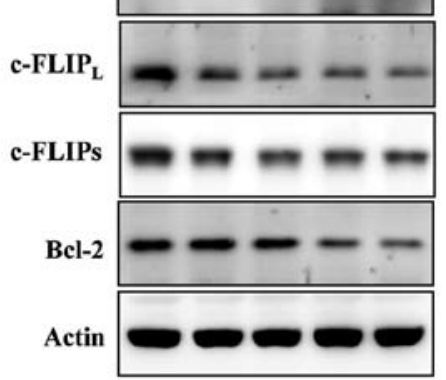

C

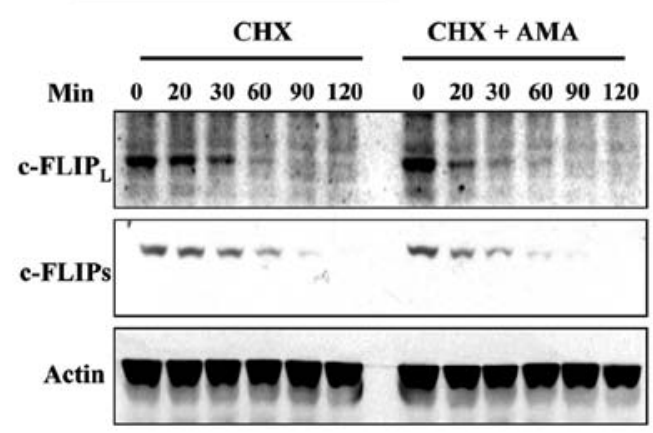

B
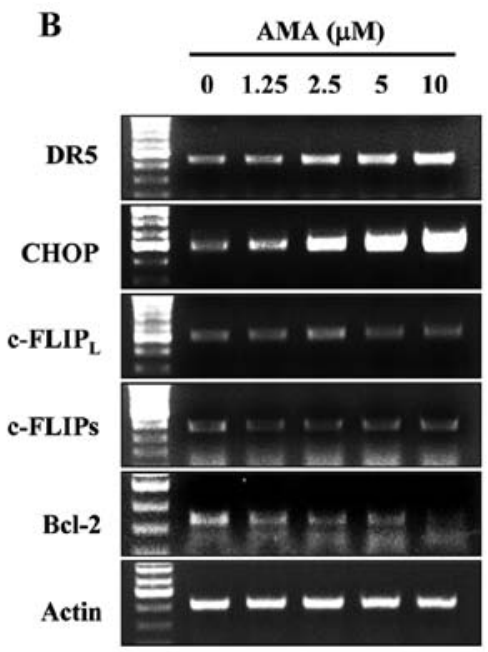

D

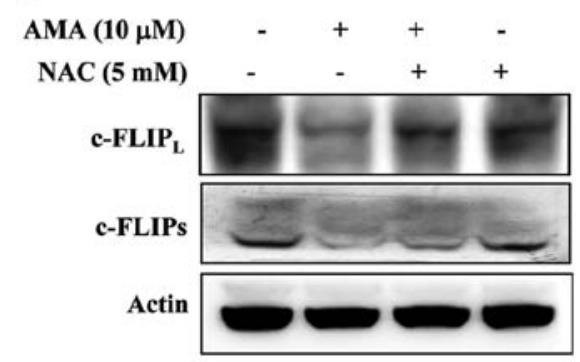

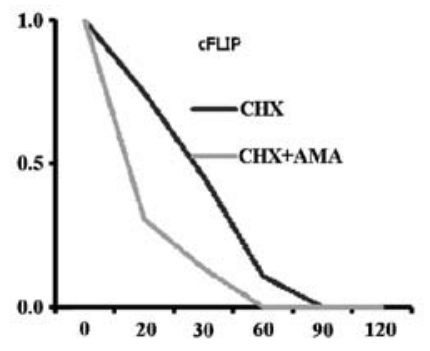

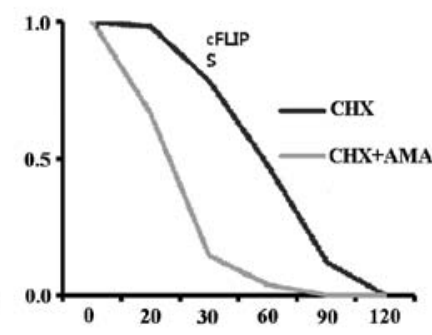

Figure 3. AMA regulates DR5 and Bcl-2 at translational level while AMA downregulates c-FLIP expressions at post-translational levels (A) Caki cells were treated with the indicated concentrations of AMA for $12 \mathrm{~h}$ and were harvested in lysis buffer. The equal amounts of cell lysates ( $40 \mu \mathrm{g})$ were resolved by SDS-PAGE. Western blot analysis was performed using anti-DR5, -CHOP, -Bcl-2, -c-FLIPs, -c-FLIPL antibodies or anti-actin antibody to serve as control for the loading of protein level. (B) Caki cells were treated with the indicated concentrations of AMA for $12 \mathrm{~h}$. Total-RNA was isolated and RT-PCR analysis was performed as described in Materials and methods. A representative study is shown; two additional experiments yielded similar results. (C) Caki cells were treated with $10 \mu \mathrm{M}$ AMA in the presence or absence of CHX for the indicated times. Western blot analysis was performed using anti-c-FLIP antibody and anti-actin antibody to serve as control for the loading of protein level. (D) Effect of NAC on protein expression induced by AMA plus TRAIL. Caki cells were incubated with $5 \mathrm{mM}$ NAC or solvent for $1 \mathrm{~h}$ before treatment with AMA $(10 \mu \mathrm{M})$ and/or TRAIL $(100 \mathrm{ng} / \mathrm{ml})$ for $12 \mathrm{~h}$. The cells were harvested in lysis buffer, and then equal amounts of cell lysates $(40 \mu \mathrm{g})$ were resolved by SDS-PAGE. Western blot analysis was performed using anti-c-FLIP antibody or anti-actin antibody to serve as control for the loading of protein level. Relative levels of each protein in CHX- or CHX plus AMA-treated cells were expressed as a fold of the densitometric value of each protein to that of control (right panel). 

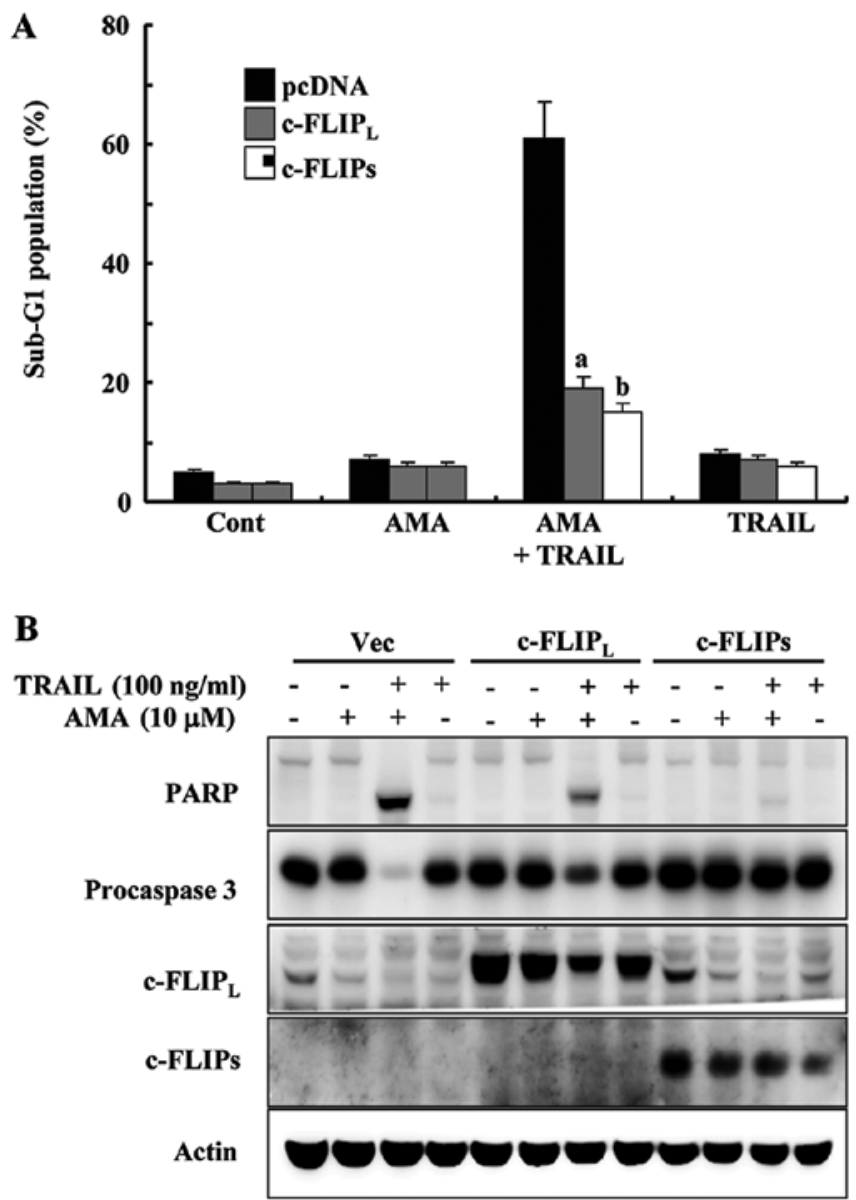

Figure 4. Downregulation of c-FLIP contributes to AMA-stimulated TRAILinduced apoptosis. (A) Caki/Vector, Caki/c-FLIPL and Caki/c-FLIPs cells were treated for $12 \mathrm{~h}$ with AMA alone $(10 \mu \mathrm{M})$, TRAIL alone $(100 \mathrm{ng} / \mathrm{ml})$ or combination of AMA and TRAIL. Apoptosis was analyzed as a sub-G1 fraction by FACS. Data shown are means $\pm \mathrm{SD}(\mathrm{n}=3){ }^{\mathrm{a}, \mathrm{b}} \mathrm{p}<0.05$ compared to AMA plus TRAIL-treated pcDNA cells. (B) Equal amounts of cell lysates $(40 \mu \mathrm{g})$ were subjected to electrophoresis and analyzed by western blotting for PARP, procaspase 3, c-FLIP $\mathrm{L}_{\mathrm{L}}$ and c-FLIPs.

level $(4,16)$ and we failed to detect that AMA-induced CHOP expression was related to DR5 upregulation (data not shown). Future studies will determine the mechanism of action of AMA treatment for upregulation of DR5 at the transcriptional level.

Several reagents such as compound C, rosiglitazone, LBH589 and silibinin can induce downregulation of c-FLIP and subsequent sensitization to TRAIL-induced apoptosis in different cancer cells (16-19). In this study, treatment with AMA induced downregulation of c-FLIP ${ }_{\mathrm{L}}$ and c-FLIPs and the enforced expression of c-FLIP ${ }_{\mathrm{L}}$ and c-FLIPs abrogated the induction of apoptosis by combination treatment with AMA and TRAIL. These results suggested a critical role of $\mathrm{c}-\mathrm{FLIP}_{\mathrm{L}}$ and c-FLIPs downregulation in mediating the augmentation of TRAIL-induced apoptosis by AMA. It is generally recognized that c-FLIP $\mathrm{L}_{\mathrm{L}}$ and c-FLIPs protein levels can be regulated by ubiquitin/proteasome mediated degradation $(20,21)$ or by their transcriptional control through the NF- $\mathrm{BB}$ or c-Fos pathway $(22,23)$. In our study, AMA promotes ubiqutin/proteasome-mediated degradation of c-FLIP ${ }_{\mathrm{L}}$ and c-FLIPs, leading to downregulation of c-FLIP, but not by transcriptional control. Recently, several studies have shown that ROS downregulates c-FLIP levels and increases the sensi-
$\mathbf{A}$

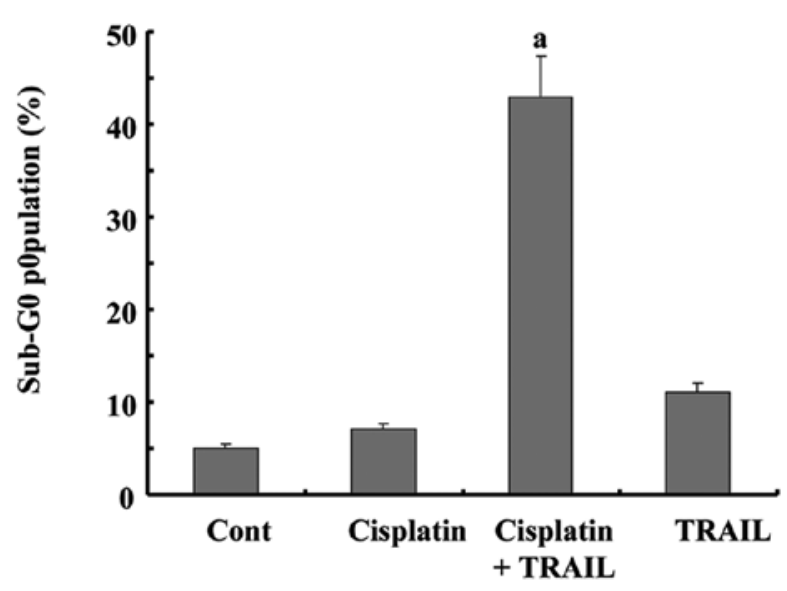

B

$\begin{array}{lllll}\text { Cisplatin }(18 \mu \mathrm{g} / \mathrm{ml}) & - & - & + & + \\ \text { AMA }(10 \mu \mathrm{M}) & - & + & + & -\end{array}$

PARP

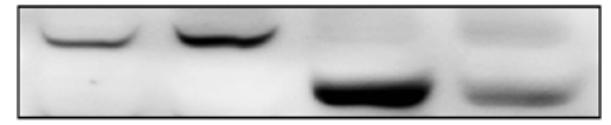

Caspase-3

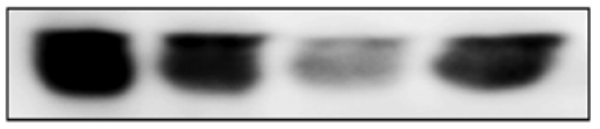

Actin

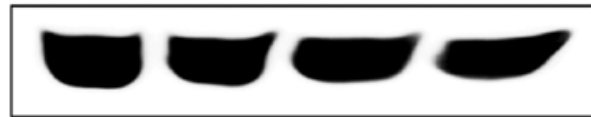

Figure 5. AMA sensitizes renal cancer cells to cisplatin-induced apoptosis (A) Caki cells were treated with cisplatin $(18 \mu \mathrm{g} / \mathrm{ml})$ in either the absence or the presence of AMA $(10 \mu \mathrm{M})$ for $12 \mathrm{~h}$. After $12 \mathrm{~h}$ treatment, apoptosis was analyzed as a sub-G1 fraction by FACS. ${ }^{\mathrm{a}} \mathrm{p}<0.05$ for AMA+cisplatin-treated cells versus control, AMA- or cisplatin-treated cells by ANOVA. (B) Cells were treated with the indicated concentrations of AMA and cisplatin. Equal amounts of cell lysates $(40 \mu \mathrm{g})$ were subjected to electrophoresis and analyzed by western blotting for PARP, procaspase 3 and actin for normalization.

tivity to apoptotic stimuli $(24,25)$. Therefore, we investigated whether downregulation of c-FLIP $\mathrm{L}_{\mathrm{L}}$ and c-FLIPs was actually mediated by ROS signaling pathway. In the presence of NAC, the decreased levels of $\mathrm{c}-\mathrm{FLIP}_{\mathrm{L}}$ and c-FLIPs caused by AMA were partly restored. Taken together, AMA-stimulated TRAILinduced apoptosis appears to be dependent on the formation of ROS for downregulations of c-FLIP ${ }_{\mathrm{L}}$ and c-FLIPs.

In summary, we suggest that AMA may be a potentially important therapeutic approach for enhancing sensitivity to TRAIL via downregulations of proteins related to the inhibition of the apoptotic processes such as Bcl-2 and c-FLIP as well as upregulation of DR5 proteins. Additionally, this study showed that AMA markedly increases sensitivity to conventional cancer chemotherapeutic agent, cisplatin, in human renal cancer Caki cells.

\section{Acknowledgements}

This research was supported by the Korea Science and Engineering Foundation (KOSEF) grant funded by the Korea government (MEST) (No. 2011-0004816). 


\section{References}

1. Pan G, Ni J, Wei YF, Yu G, Gentz R and Dixit VM: An antagonist decoy receptor and a death domain-containing receptor for TRAIL. Science 277: 815-818, 1997.

2. Sheridan JP, Marsters SA, Pitti RM, et al: Control of TRAIL-induced apoptosis by a family of signaling and decoy receptors. Science 277: 818-821, 1997.

3. Ivanov VN, Partridge MA, Johnson GE, Huang SX, Zhou H and Hei TK: Resveratrol sensitizes melanomas to TRAIL through modulation of antiapoptotic gene expression. Exp Cell Res 314: 1163-1176, 2008.

4. Lee TJ, Um HJ, Min do S, Park JW, Choi KS and Kwon TK: Withaferin A sensitizes TRAIL-induced apoptosis through reactive oxygen species-mediated up-regulation of death receptor 5 and down-regulation of c-FLIP. Free Radic Biol Med 46: 1639-1649, 2009.

5. Jung EM, Lim JH, Lee TJ, Park JW, Choi KS and Kwon TK: Curcumin sensitizes tumor necrosis factor-related apoptosisinducing ligand (TRAIL)-induced apoptosis through reactive oxygen species-mediated upregulation of death receptor 5 (DR5). Carcinogenesis 26: 1905-1913, 2005.

6. You BR and Park WH: The effects of antimycin A on endothelial cells in cell death, reactive oxygen species and GSH levels. Toxicol In Vitro 24: 1111-1118, 2010.

7. Cai J and Jones DP: Superoxide in apoptosis. Mitochondrial generation triggered by cytochrome c loss. J Biol Chem 273: 11401-1144, 1998.

8. Pham NA, Robinson BH and Hedley DW: Simultaneous detection of mitochondrial respiratory chain activity and reactive oxygen in digitonin-permeabilized cells using flow cytometry. Cytometry 41: 245-251, 2000.

9. Turrens JF: Superoxide production by the mitochondrial respiratory chain. Biosci Rep 17: 3-8, 1997.

10. Pastorino JG, Tafani M, Rothman RJ, Marcinkeviciute A, Hoek JB and Farber JL: Functional consequences of the sustained or transient activation by Bax of the mitochondrial permeability transition pore. J Biol Chem 274: 31734-31739, 1999.

11. Petronilli V, Penzo D, Scorrano L, Bernardi P and Di Lisa F: The mitochondrial permeability transition, release of cytochrome $\mathrm{c}$ and cell death. Correlation with the duration of pore openings in situ. J Biol Chem 276: 12030-12034, 2001.

12. Lee TJ, Jung EM, Lee JT, et al: Mithramycin A sensitizes cancer cells to TRAIL-mediated apoptosis by down-regulation of XIAP gene promoter through Sp1 sites. Mol Cancer Ther 5 2737-2746, 2006.

13. Moon DO, Kim MO, Choi YH and Kim GY: Butein sensitizes human hepatoma cells to TRAIL-induced apoptosis via extracellular signal-regulated kinase/Sp1-dependent DR5 upregulation and NF-kappaB inactivation. Mol Cancer Ther 9: 1583-1595, 2010.
14. Frese-Schaper M, Schardt JA, Sakai T, Carboni GL, Schmid RA and Frese S: Inhibition of tissue transglutaminase sensitizes TRAIL-resistant lung cancer cells through upregulation of death receptor 5. FEBS Lett 584: 2867-2871, 2010.

15. Fan S, Li Y, Yue P, Khuri FR and Sun SY: The eIF4E/eIF4G interaction inhibitor 4EGI-1 augments TRAIL-mediated apoptosis through c-FLIP down-regulation and DR5 induction independent of inhibition of cap-dependent protein translation. Neoplasia 12: 346-356, 2010.

16. Son YG, Kim EH, Kim JY, et al: Silibinin sensitizes human glioma cells to TRAIL-mediated apoptosis via DR5 up-regulation and down-regulation of c-FLIP and survivin. Cancer Res 67: 8274-8284, 2007.

17. Kim YH, Jung EM, Lee TJ, et al: Rosiglitazone promotes tumor necrosis factor-related apoptosis-inducing ligand-induced apoptosis by reactive oxygen species-mediated up-regulation of death receptor 5 and down-regulation of c-FLIP. Free Radic Biol Med 44: 1055-1068, 2008.

18. Jang JH, Lee TJ, Yang ES, et al: Compound C sensitizes Caki renal cancer cells to TRAIL-induced apoptosis through reactive oxygen species-mediated down-regulation of c-FLIPL and Mcl-1. Exp Cell Res 316: 2194-2203, 2010.

19. Kauh J, Fan S, Xia M, et al: c-FLIP degradation mediates sensitization of pancreatic cancer cells to TRAIL-induced apoptosis by the histone deacetylase inhibitor LBH589. PLoS One 5: e10376, 2010.

20. Poukkula M, Kaunisto A, Hietakangas V, et al: Rapid turnover of c-FLIPshort is determined by its unique C-terminal tail. J Biol Chem 280: 27345-27355, 2005.

21. Kaunisto A, Kochin V, Asaoka T, et al: PKC-mediated phosphorylation regulates c-FLIP ubiquitylation and stability. Cell Death Differ 16: 1215-1226, 2009.

22. Li W, Zhang X and Olumi AF: MG-132 sensitizes TRAILresistant prostate cancer cells by activating c-Fos/c-Jun heterodimers and repressing c-FLIP(L). Cancer Res 67: 2247-2255, 2007.

23. Benayoun B, Baghdiguian S, Lajmanovich A, et al: NF-kappaBdependent expression of the antiapoptotic factor c-FLIP is regulated by calpain 3 , the protein involved in limb-girdle muscular dystrophy type 2A. FASEB J 22: 1521-1529, 2008.

24. Nitobe J, Yamaguchi S, Okuyama M, et al: Reactive oxygen species regulate FLICE inhibitory protein (FLIP) and susceptibility to Fas-mediated apoptosis in cardiac myocytes. Cardiovasc Res 57: 119-128, 2003.

25. Kanayama A and Miyamoto Y: Apoptosis triggered by phagocytosis-related oxidative stress through FLIPS down-regulation and JNK activation. J Leukoc Biol 82: 1344-1352, 2007. 